EPiC Series in Computing
Volume 70, 2020, Pages 217-225
$\begin{gathered}\text { Proceedings of the 12th International Conference } \\ \text { on Bioinformatics and Computational Biology }\end{gathered}$

\title{
Hepatocyte Organization Affects the Translation of Clearance from In Vitro to In Vivo
}

\author{
Lopamudra Dutta ${ }^{1}$, Preethi Krishnan ${ }^{1}$, Andrew K. Smith ${ }^{1}$, \\ Ryan C. Kennedy ${ }^{1}$, Glen E. P Ropella ${ }^{2}$, and C. Anthony Hunt ${ }^{1 *}$ \\ ${ }^{1}$ Department of Bioengineering and Therapeutic Sciences, University of California, San \\ Francisco, CA, 94143 \\ ${ }^{2}$ Tempus Dictum, Inc., Milwaukie, OR 97222 \\ lopamudra2008@yahoo.com, preethikrishnan27@gmail.com, \\ drandrewksmithegmail.com, ryan.c.kennedyealumni.nd.edu, \\ gepr@tempusdictum.com, a.hunteucsf.edu
}

\begin{abstract}
An improved understanding of in vivo $\Leftrightarrow$ in vitro changes is crucial in identifying and mitigating factors contributing to in vitro-in vivo extrapolation (IVIVE) inaccuracies in predicting the hepatic clearance of substances. We argue that a model mechanism-based virtual culture (vCulture) $\Leftrightarrow$ virtual mouse (vMouse) (or vRat or vHuman) experiment approach can identify factors contributing to IVIVE disconnects. Doing so depends on having evidence that six Translational Requirements have been achieved. We cite evidence that the first four have been achieved. The fifth Requirement is that differences in measures of vCompound disposition between vCulture and vMouse are attributable solely to the micro-architectural, physiomimetic features, and uncertainties built into the vLiver and vMouse but are absent from the vCulture. The objective of this work is to first improve on a vCulture architecture used previously and then use results of virtual experiments to verify that its use enables the fifth Translational Requirement to be achieved. We employ two different idealized vCompounds, which map to highly permeable real compounds at the extreme ends of the intrinsic clearance spectrum. Virtual intrinsic clearance $=$ Exposure rate per vHPC. At quasi-steady state, results for vCompound-1 are independent of the dosing rate. The average per-vHPC Exposure rates (taken over the whole vLiver in vMouse experiments) are the same (within the variance of the Experiments) as those in vCulture. However, they are location dependent within the vLiver. For vCompound-2, there are dosing rate differences and average per-vHPC Exposure rates within the vLiver are also location dependent. When we account for dosing rate differences, we see again that average per-vHPC Exposure rates averaged over the whole vLiver in vMouse experiments are the same as those in vCulture. Thus, the differences in per vHPC Exposure rate within the vLiver for both vCompounds are attributable solely to
\end{abstract}


Hepatocyte Organization Affects the Translation of Clearance from In Vitro to In Vivo Dutta et al.

the micro-architectural and physiomimetic features built into the vLiver and vMouse but are absent from the vCulture. The results verify that the fifth Translational Requirement has been achieved.

\section{Introduction}

In vitro-to-in vivo extrapolation (IVIVE) methods for predicting the hepatic clearance of substances continue to improve. They still often underpredict in vivo results by $>40 \%$, and two-fold differences and larger are common [1]. The reasons are still poorly understood. Nevertheless, it is well-understood that a major cause of these IVIVE disconnects is entanglement of different methodological and biological factors [2]. Smith et al. (2018) suggest identifying factors contributing to and subsequently bridging IVIVE disconnects by instantiating then experimenting on concrete and scientifically challengeable model Mechanisms within validated virtual culture and virtual mouse contexts [3]. To distinguish virtual mouse and culture components, characteristics, and phenomena from real counterparts, we capitalize the former hereafter and, in several cases, append the prefix " $v$ ".

A conventional IVIVE begins by measuring the depletion of a compound in vitro using microsomes or hepatocytes and then calculating a value of in vitro intrinsic clearance. This work focuses on hepatocyte (HPC) cultures. The calculated intrinsic clearance per HPC is scaled to a whole liver in the target organism to provide an estimate of in vivo intrinsic clearance from which a corresponding species-specific in vivo hepatic clearance can be calculated using an estimate of hepatic blood flow and a mathematical hepatic disposition model. A core operating hypothesis is that, on average, the intra-HPC mechanisms responsible for compound clearance, via metabolism and biliary elimination, are the same in vivo as in vitro. A necessary assumption is that, under quasisteady state conditions, the compound's availability to HPCs (exposure, defined below) is essentially the same in vivo as in vitro.

An improved understanding of in vivo $\Leftrightarrow$ in vitro changes is crucial to interpreting in vitro data correctly and further improving hepatocyte-based IVIVEs to human targets. We argue that the vCulture $\Leftrightarrow$ vMouse approach described by Smith et al. (2018) can be used to identify and mitigate factors contributing to IVIVE inaccuracies in predicting the hepatic clearance of substances [3]. Credible applications of that approach will depend on evidence demonstrating that six translational requirements have been achieved. Translational Requirements 1-4 are provided in Methods, and the evidence cited supports that they have been achieved. The objective of this work is to improve the cited vCulture architecture and verify that the following Translational Requirement 5 is achieved.

Translational Requirement 5: (vCulture $\Leftrightarrow$ vMouse translations) For comparable Experimental conditions employing the same number of identically parameterized vHPCs, the differences in measures of vCompound disposition between vCulture and vMouse are attributable to the microarchitectural, physiomimetic features, and uncertainties built into the vLiver and vMouse but are absent from the vCulture. Verification of Translational Requirement 5 is a requisite for future validation experiments (Translational Requirement 6) demonstrating that vPharmacokinetic measures can be scaled directly to provide model mechanism-based predictions of in vivo clearance.

We employ two different idealized vCompounds, which map to highly permeable real compounds. vCompound-1 is located at the low-value end of the intrinsic clearance spectrum. vCompound-2 enters but does not exit vHPCs and is located at the high-value end of the intrinsic clearance spectrum. We record each vCompound-vHPC entry event, and define it as an Exposure event. Results for vCompound-1 show that at quasi-steady state, independent of vCompound-1 delivery rate, the average per-vHPC Exposure rates in both vLiver and vCulture Experiments are the same (within the variance of the Experiments). Nevertheless, vCompound-1 Exposure rates are location dependent within the vLiver. Those differences are attributable solely to the micro- 
architectural and physiomimetic features built into the vLiver and vMouse but are absent from the vCulture.

For the same amount of vCompound-2, Exposure rate per vHPC equals virtual intrinsic clearance. Results show that the per-vHPC Exposure rates in vCulture and vLiver are dependent on the rate of vCompound-2 delivery to vHPCs. However, the Exposure rate differences between the two systems, which are evident at the vMouse level for vCompound-2, are not evident at the whole vLiver level, yet they too are location dependent within the vLiver. Again, the latter differences are attributable solely the physiomimetic features built into the vLiver and vMouse. Taken together, the results from Experiments verify that Translational Requirement 5 has been achieved.

\section{Methods}

\subsection{Requisite Mechanism Requirements}

During execution the mechanism responsible for a vCompound's disposition must exhibit the five characteristics of a biologically explanatory mechanism [4].

1. The mechanism is biomimetic and responsible for the phenomena.

2. The mechanism has components: modules, entities, and activities.

3. Components are arranged spatially and can exhibit structure, localization, orientation, connectivity, and compartmentalization.

4. Activities have temporal aspects, including rate, order, duration, and frequency.

5. The mechanism has a context, which can include being in a series and/or a hierarchy.

\subsection{Requisite Translational Requirements}

1. In vitro culture $\Leftrightarrow$ vCulture: instantiate a vCulture containing vHPCs. Make it strongly analogous to an actual HPC culture used to measure the intrinsic clearance of real compounds. Use an iterative refinement process, which includes model mechanism falsification and validation to discover a parameterization of the vCulture, vHPCs, and a vCompound such that the vCompound's Intrinsic Clearance maps quantitatively to intrinsic clearance.

2. HPCs $\Leftrightarrow$ vHPCs: develop evidence that the disposition mechanisms in (1) are concrete and biomimetic. Petersen et al. (2016) provides evidence supporting (1) and (2) [5].

3. Mammalian liver $\Leftrightarrow$ vLiver: using the iterative refinement process in (1), compile quantitative evidence that the concrete quasi-3D structure of a vLiver is strongly analogous to the actual 3D structure of a mammalian liver. Yan et al. (2008) provide evidence supporting the strength of the analogy [6], and Smith et al. (2016) provide evidence that strengthens the analogy [7].

4. vLiver in vMouse $\Leftrightarrow$ mouse liver pharmacodynamics: measures of the spatial Pharmacodynamics of a vCompound within a vLiver explain (within some tolerance) hepatic pharmacodynamic measures of a real drug in mice. Smith et al. (2016) do so [7].

\subsection{Simulation Details}

Experiments were conducted using a vLiver within a vMice that were created using agent oriented modelling techniques and the Java-based MASON multiagent toolkit. Doing so allows the vLiver to be biomimetic in multiple ways, such as having Cell objects respond to external and internal stimuli. The vLiver and its components are biomimetic analogies. Their concreteness allows their features to be measured and annotated Mappings - qualitative and quantitative - can be established between virtual and biological features and measures. Modular vLiver components can be reused for 
different experiment use cases and versioning allows one component to evolve somewhat independent of others. Experiments are used to challenge model mechanism-based hypotheses, support the veracity of claims made, and to explore the behavior spaces of model mechanism variants. Experiments are executed using Google Compute Engine running Linux. The R programming language is used for data analyses. The entire toolchain, including the operating system, configurations, and I/O handling is open-source. The data presented herein, along with the code, are available from project websites (https://simtk.org/projects/aili and https://simtk.org/home/isl/), and are available to be licensed as open data.

\subsection{Virtual Liver and Virtual Components}

Verification of Translational Requirement 5 necessitated improving the vCulture architectures used previously [3,5]. To improve the credibility of current and future vCulture $\Leftrightarrow$ vMouse translation experiments, we also made the vMouse architecture more physiomimetic. In the cited versions of the vCulture architecture, the Dose was transferred directly to the single graph Layer. In the current version, the Body space in a vMouse in repurposed as the Media Space in a vCulture, and a vLobule's PV, CV, and Sinusoidal Segment (SS) spaces are repurposed to mimic a "static diffusion layer" between HPCs and the culture media. These improvements reduce software complexity and allow for more flexible, biomimetic parameterizations. Previous vLobule variants used a 3-Layer directed graph structure. We transitioned to a 5-Layer vLobule architecture to enable a more physiomimetic mapping to the complex flow paths within actual hepatic lobules.

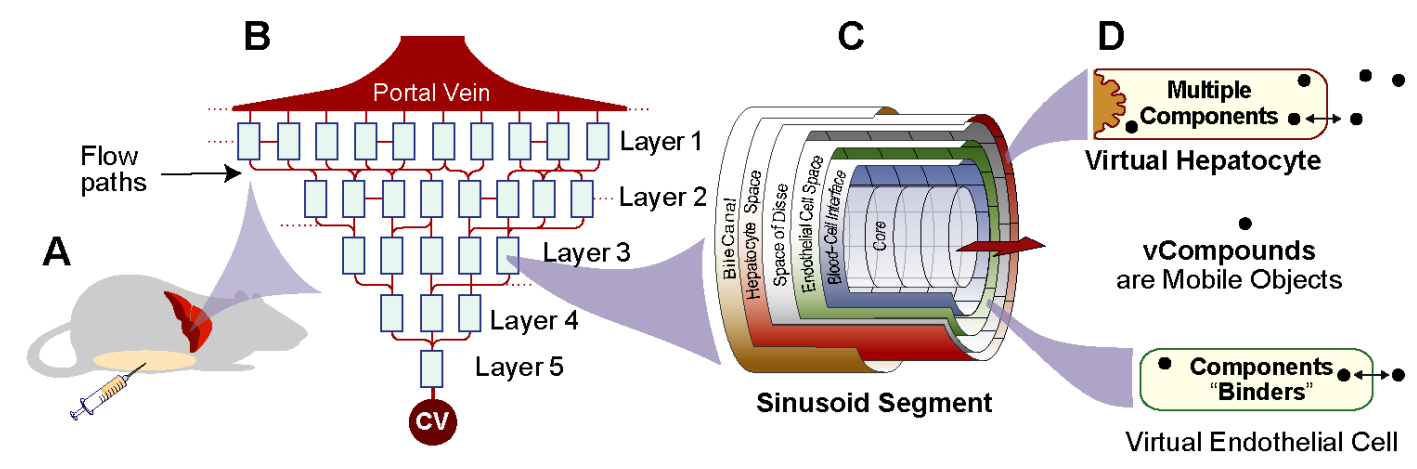

Figure 1. Virtual Mouse components. (A) A vMouse comprises a vLiver (a Monte Carlo-sampled number of vLobules), Mouse Body, a space to contain Dose, and a process that transfers vCompounds from Mouse Body to vLiver. Measurements of vCompound in Mouse Body map to referent drug plasma levels. (B) A portion of vLobule is illustrated. Red colored graph edges indicate flow connections within and between five Layers. (C) A multi-layered, quasi-3D SS maps to a portion of lobular tissue. An SS functions during execution analogous to sinusoid components and features averaged across many actual lobules; SS dimensions are Monte Carlo (MC)sampled to mimic lobular variability. An SS comprises a Core surrounded concentrically by five $2 \mathrm{D}$ grids. Mobile vCompound objects move within and between these grids. vCompounds enter and exit an SS via Core and Interface, percolate stochastically through accessible spaces influenced by configuration-controlled local flow parameters. vCompounds that exit to the CV are returned to Mouse Body. (D) Each vHPC and Endothelial Cell controls vCompound entry from and exit to adjacent spaces, and the fate of vCompounds within.

Because the vLiver is a concretized biomimetic analogy of an actual liver, features do not map 1:1 to biological counterparts. A vLiver is a specified number of Monte Carlo (MC)-sampled vLobule 
variants. A vLobule (Fig. 1) maps to a small random sample of possible lobular flow paths within a whole liver and to the total volume of associated tissue. A SS does not map directly to a portion of a single sinusoid surrounded by hepatic endothelial cells, hepatocytes, etc. However, events occurring within an SS are intended to be strongly analogous to referent events within portions of sinusoids and adjacent tissue [8]. A vHPC maps to a conflation of relevant hepatocyte functionality that a small fraction dose encounters at a corresponding relative PV-to-CV location.

vLobules are designed so that vCompounds entering PV get exposed to a decreasing number of hepatocytes as they approach the CV. A vLobule is a directed acyclic graph with an SS agent at each node. The graph nodes are organized into five Layers. Previous vLobules were organized into three Layers. To reflect the polyhedral nature of hepatic lobules [9], the number of nodes in Layers 1-5 are 45, 25, 20, 14, and 9, respectively. An SS (Fig. 1C) comprises a Core, Blood-Cell Interface, Endothelial Cell Space, Space of Disse, Hepatocyte Space, and Bile Space (not used during this study). Cell objects are software agents which occupy 99\% of Endothelial Cell and all Hepatocyte Spaces.

\section{5 vCompounds}

A vCompound is a mobile object. In this work, for all Experiments, the Dose of vCompounds is 50,000 objects. When a vCompound has a real-world counterpart, e.g., acetaminophen [7], a vCompound maps to a small fraction of a referent drug dose. Cells recognize vCompounds and adjust their responses appropriately during each simulation cycle. For example, a vHPC recognizes that an adjacent vCompound outside the Cell has the property membrane crossing = true, and, with a specified probability, allows it to enter. A vCompound's vHPC Entry and Exit probability (designated pEnter and pExit) is mediated by each Cell. In this work, pEnter $=1.0$ for both vCompounds, which maps to real compounds having an unbound fraction $=1.0$. A vHPC recognizes that an unbound vCompound within has an assigned probability of exiting, pExit, and, each cycle, determines if an Exit will (or not) occur. For vCompound-1, $p$ Exit $=1.0$. For vCompound -2 , $p E x i t=0$. The dynamics of vCompound-1 within vLobules and vCultures provides a plausible representation of corresponding dynamics of highly permeable real compounds located at the low-value end of the intrinsic clearance spectrum. The dynamics of vCompound-2 within vLobules and vCultures provides a plausible representation of the dynamics of corresponding highly permeable real compounds located at the high-value end of the intrinsic clearance spectrum.

All Cells contain a probability-specified number of Binders $(\operatorname{Max}=10$, Min $=5)$. The actual number for each Cell is determined by $\mathrm{MC}$ sampling at the start of an execution. Binders within vHPCs can be parameterized to Metabolize a Bound vCompound, but that is not done in this work. However, with probability $=0.1$, both vCompounds can be Bound by vHPC Binders to simulate a small degree of non-specific binding. A Bound vCompound is released after 10 simulation cycles.

During each simulation cycle, a fraction of vCompound in Mouse Body is transferred to PV. From PV, vCompounds enter Core and Interface space at the upstream end of all Layer-1 SSes and then percolate stochastically toward Hepatocyte Space and the CV [6], influenced by local flow specifications. They exit the last SS, enter the CV, and then get moved to Mouse Body. Intra-Lobular vCompound movement is parameter-controlled and is the same for vCompounds- 1 and -2 . The simulated blood flow rate in Core is specified by ssFlowRate. Outside the Core, extra-Cellular vCompound movement is a biased random walk. Forward and lateral movement is controlled by forwardBias and lateralBias, which can be specified separately for Core/Interface and the outer spaces (Endothelial, Space of Disse, etc.). 


\subsection{Virtual Culture}

Petersen et al. (2016) used an early version of a vCulture to explore and challenge mechanismbased hypotheses about immune-mediated P450 down-regulation in vitro [5]. Smith et al. (2018) used and updated, more biomimetic vCulture to demonstrate describe a virtual counterpart of an in vivo-in vitro disconnect [3]. For this work we use the enhanced version of their vCulture illustrated in Fig. 2. The vCulture reuses all vHPCs from a vMouse experiment. vCulture does not use the vLobule's directed graph. All vHPCs from each SS are reinstantiated and placed into a corresponding vCulture SS Space. A vCulture does not use Core and Bile spaces, nor the Endothelial Cell and Disse grids. It does reuse the Interface grid. We remap the latter as the Media-Cell interface. Each simulation cycle, a small fraction of vCompound in Media is transferred randomly to each Media-Cell Interface grid. From there vCompounds move within Hepatocyte Space and enter and exit vHPCs exactly as they do within vLobules.

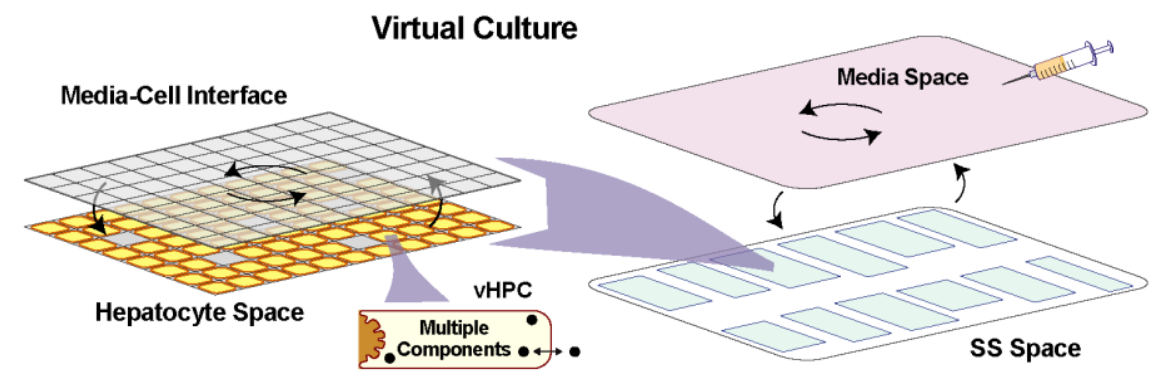

Figure. 2. Virtual Culture. A vCulture is a variant of a vLobule. All vLobule SSs are simplified and placed in an SS space. All vLobule vHPCs are reused. vHPC configurations within the vLiver and the vCulture are identical. The vMouse Body space becomes the vCulture's well-mixed Media Space. The Hepatocyte Space is retained, but the Epithelial Cell Space is removed. All other spaces are merged into a Media-Cell Interface. vCompounds move identically within and between vLobule and vCulture spaces.

\subsection{Measurements}

Results of Experiments help us understand and document how changes in pEnter and pExit impact vCompound dynamics within SSes. Measurements of various features are made analogous to wet-lab counterparts to facilitate comparisons when wet-lab data are available. Because each execution's detailed specifications are determined using MC sampling, and the unfolding of events is stochastic, there can be considerable variability among measures from multiple executions. To aid verification, we also count the number of vCompounds in several locations: 1) within Mouse Body, 2) extraCellular within Endothelial Cell Space, Space of Disse, or Hepatocyte Space, but not within the cell agents, 3) intra-Cellular within vHPCs or 4) elsewhere.

We define a vHPC Exposure event as one Entry event. We record each vHPC Exposure event. Exposure rate $=$ Exposure events per cycle averaged over the vHPCs measured. During vCulture Experiments, Exposure rates for all vHPCs are indistinguishable, within MC variance. During vMouse Experiments, Exposure rates within the vLiver can be location dependent. To document that dependency, we measure Exposure rates within the Periportal and Pericentral bands illustrated in Fig. 2 , and record the average value per vHPC for each band. To facilitate mappings to in vivo measures, we also record average Exposure rates for each vLobule. Under quasi-steady state conditions, the Intrinsic Clearance per vHPC per cycle $=$ Exposure events - Exit events. The vLiver extraction ratio each cycle is defined as $1-$ [vCompound count that exited]/[vCompound count that entered]. 


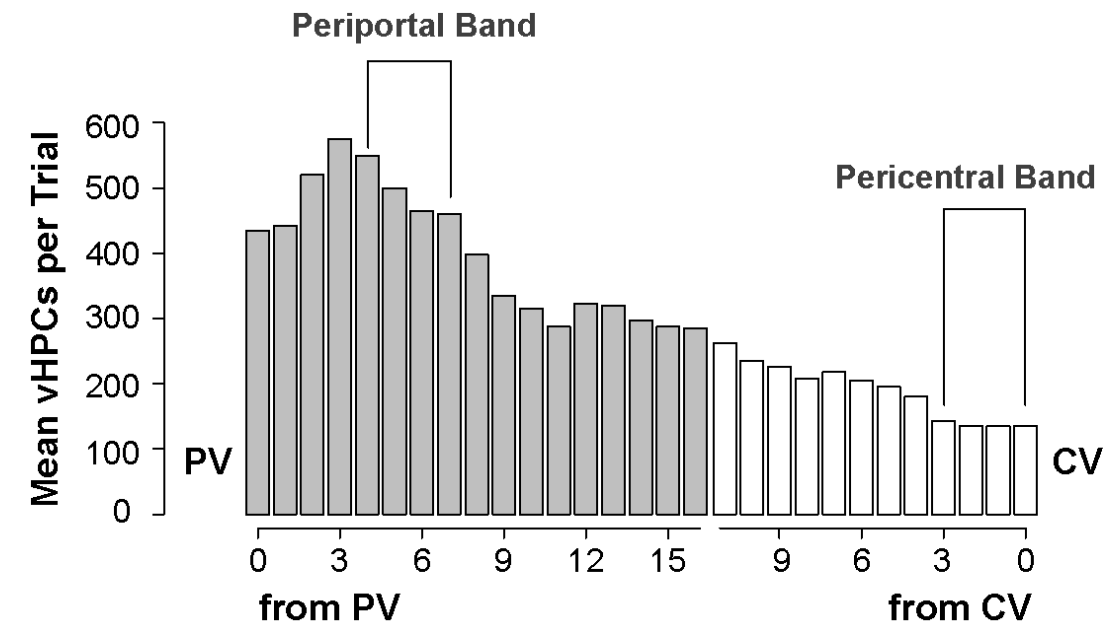

Figure. 3. Mean number of vHPCs at each PV-to-CV location within an average vLiver. Dark gray bars (spanning Layers-1-3 and part of Layer-4 in Fig. 1) are mean number of vHPCs at grid points 0-16 (ordinal numbering) from the PV (designated dPV). White bars (spanning Layer-5 and part of Layer-4) are mean numbers at grid points $0-13$ from the $\mathrm{CV}$ (designated $\mathrm{dCV}$ ). Both $\mathrm{dPV}$ and $\mathrm{dCV}$ measures are needed because the shortest and longest PV-to-CV paths can be different for each execution. SS lengths in Layer 5 are fixed. Measures are recorded within a Periportal band ( $\mathrm{dPV}=4-7)$ and a Pericentral band $(\mathrm{dCV}=0-3)$.

\section{Results and Discussion}

Results are presented from two vMouse and four vCulture experiments, each using $6 \mathrm{MC}$ trials. For the two vMouse experiments, the first order "absorption" into Body and the transfer rate from Body to PV (maps to hepatic blood flow) are the same as in Smith et al. $(2016,2018)$ [7, 3]. At the start of vCulture experiments, the Dose is introduced to Media Space using a rapid and prolonged process. Rapid addition (followed by simulated rapid mixing) maps to an idealized addition of compound to culture media. For the prolonged addition, the Dose was introduced to Media Space using an input rate that enabled the timing for achieving quasi-steady state Exposure Rate for vCompound-1 to be approximately the same as in the vMouse experiment, which facilitates comparisons of vMouse and vCulture measures.

Calculated Exposure Rates (Exposure events per cycle averaged over the vHPCs measure) for vCompound-1 and -2 are presented in Fig. 4. Within the variance of 6 MC trials using vCompound-1, the quasi-steady state Exposure Rates are the same for vCulture and vMouse experiments, which verifies that, with the improved the vCulture architecture, the fifth requirement criteria is achieved. At quasi-steady state, the disposition of vCompound- 1 within the different vCulture and vMouse spaces, along with average access to vHPCs are identical, independent of the Dose input rates used. Thus, any differences in vCompound disposition between the two systems are attributable to the microarchitectural and physiomimetic features built into the vLiver and vMouse. The differences between the two systems for vCompound-1 are not evident at the whole vLiver level, yet they are strikingly evident when Exposure Rates within the two bands are compared. Within the Pericentral band (Fig. 4B) Exposure Rates are 3.7-fold larger than within the Periportal band.

The results in Fig. 4C show that vCulture-vMouse Exposure Rate for vCompounds-2 (Fig. 4C) are different. In the vCulture, each cycle, Exposure Rate per vHPC is a function of vCompound-2 delivery to Media-Cell Interface Space. To match the Exposure Rate in the vMouse experiments, the 
prolonged addition rate to Media Space can be reduced further. As with vCompound-1, the differences between the two systems for vCompound-2 are not evident at the whole vLiver level, yet again, they are evident when Exposure Rates within the two bands are compared (Fig. 4D). Within the Pericentral band, Exposure Rates at $60 \mathrm{~min}$. are 1.3-fold larger than within the Periportal band, even though there is considerable removal of vCompound-2 by upstream vHPCs. That difference is attributable to the micro-architectural and physiomimetic features built into the vLiver and vMouse. Taken together, the results in Fig. 4 verify that, for the conditions specified, the fifth Translational Requirement is achieved.
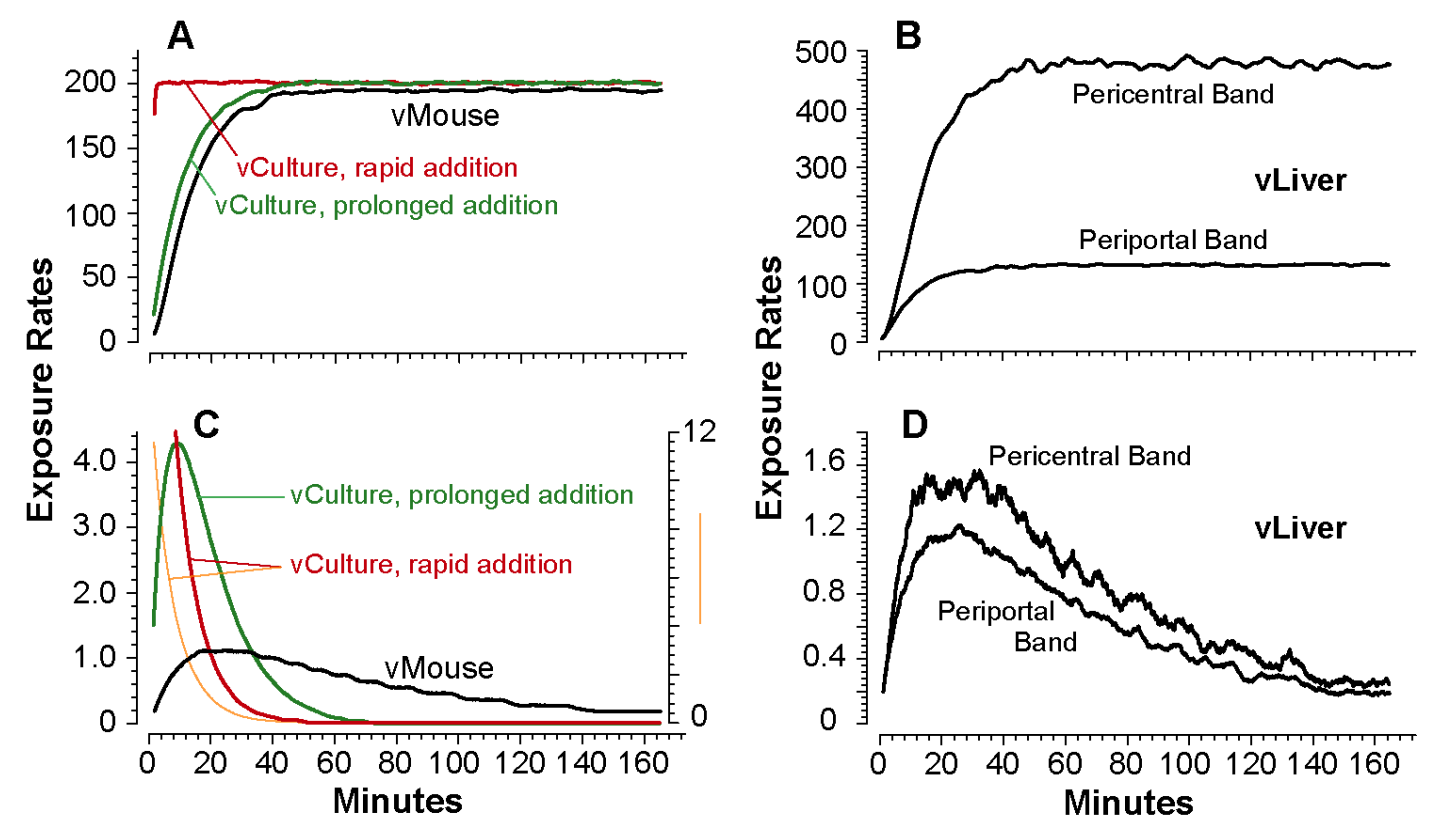

Figure 4. Exposure Rates for vCompounds-1 and -2. Plotted values are centered moving averages spanning 181 cycles. (A) vCompounds-1 Exposure Rates during vCulture and vMouse experiments. (B) vCompounds-1 Exposure Rates within the two Fig. 3 bands during the vMouse experiment. (C) vCompounds-2 Exposure Rates during vCulture and vMouse experiments. The orange profile (right axis) includes all vCompounds-2 measurements. (D) vCompounds-2 Exposure Rates measured within the two Fig. 3 bands during the vMouse experiment.

Disconnect evidence from vCulture $\Leftrightarrow$ vHPCs experiments can stand as a concrete, challengeable theory explaining how loss of lobular micro-architectural and physiological features during HPC isolation has contributed to particular in vivo-in vitro disconnects. An abridged description of the approach to do so is illustrated in Fig. 5. The vMouse and vCulture systems independently build credibility through cumulative achievement of qualitative and quantitative validation targets with their real counterparts (bottom in Fig. 5). Differences in phenomena measured during vCulture experiments and corresponding measurements of phenomena recorded during vMouse experiments are entirely explainable: there is no disconnect. Model mechanism differences that explain vMouse $\Leftrightarrow$ vCulture translations can stand as a concrete, scientifically challengeable hypothesis that explain (or not) observed disconnects in their real counterparts. The concretized vMouse $\Leftrightarrow$ vCulture theory provides the framework to develop more reliable interpretations of in vitro observations, which then may be used to improve extrapolations. 
Hepatocyte Organization Affects the Translation of Clearance from In Vitro to In Vivo Dutta et al.

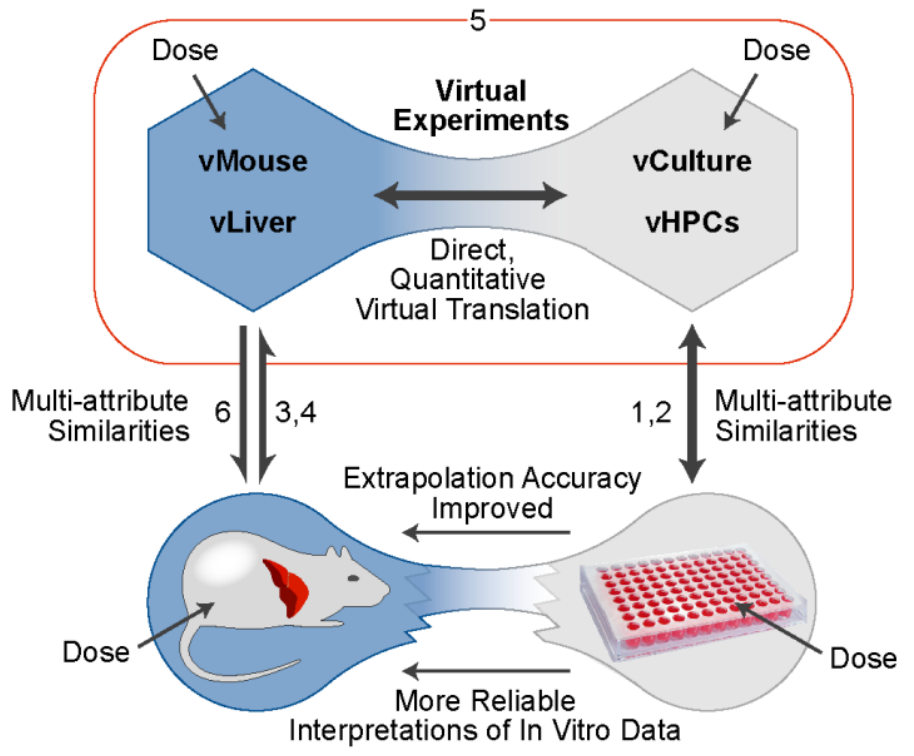

Figure 5. An approach to bridge IVIVE disconnects [3]. The numbers refer to the Translational Requirements enumerated in the Introduction and Method.

\section{References}

[1] Bowman CM and Benet LZ. Hepatic clearance predictions from in vitro-in vivo extrapolation and BDDCS. Drug Metab Dispos. 2016;44:1731-35.

[2] Hallifax D, Foster JA, and Houston JB. Prediction of human metabolic clearance from in vitro systems: retrospective analysis and prospective view. Pharm Res. 2010;27:2150-61.

[3] Smith AK, Xu Y, Ropella GEP, and Hunt CA. A Model Mechanism-Based Explanation of an In Vitro-In Vivo Disconnect for Improving Extrapolation and Translation. J Pharmacol Exp Ther 2018;365:127-38.

[4] Darden L. Thinking again about biological mechanisms. Philos Sci. 2008;75:958-69.

[5] Petersen BK, Ropella GE, and Hunt CA. Virtual experiments enable exploring and challenging explanatory mechanisms of immune-mediated P450 down-regulation. PloS ONE. 2016;11:e 0155855.

[6] Yan L, Ropella GE, Park S, Roberts MS, and Hunt CA. Modeling and simulation of hepatic drug disposition using a physiologically based, multi-agent in silico liver. Pharm Res. 2008;25:1023-36.

[7] Smith AK, Petersen BK, Ropella GE, Kennedy RC, Kaplowitz N, Ookhtens M, and Hunt CA. Competing mechanistic hypotheses of acetaminophen-induced hepatotoxicity challenged by virtual experiments. PLoS Comput Biol. 2016;12:e1005253.

[8]Hunt CA, Ropella GE, Lam TN, and Gewitz AD. Relational grounding facilitates development of scientifically useful multiscale models. Theor Biol Med Model. 2011;8:35-66.

[9] Teutsch HF, Schuerfeld D, and Groezinger E. Three-dimensional reconstruction of parenchymal units in the liver of the rat. Hepatology. 1999;29:494-505. 